\title{
ACLIMATAÇÃO E FISIOLOGIA DE MUDAS DE GUANANDI CULTIVADAS EM TELAS DE SOMBREAMENTO FOTO-PROTETORAS
}

\author{
Gustavo F. R. Saraiva ${ }^{1}$, Gustavo Maia Souza ${ }^{1}$, João Domingues Rodrigues ${ }^{2}$ \\ ${ }^{1}$ Universidade do Oeste Paulista - UNOESTE, Programa de Pós-Graduação em Agronomia, Laboratório de Inteligência em Plantas e Ecofisiologia \\ "Ulrich Luttge" - LIPEUL, Presidente Prudente - SP. Universidade Estadual Paulista “Júlio de Mesquita Filho" - UNESP. Pós-graduação em Botânica, \\ Botucatu - SP. E-mail: saraivaunoeste@hotmail.com, mingo@ibb.unesp.br
}

\section{RESUMO}

A luminosidade é o fator mais importante no crescimento e desenvolvimento de plântulas. As plantas modificam seu modelo de crescimento e alocação de recursos em resposta ao ambiente luminoso. Foram avaliados parâmetros biométricos, bioquímicos e fisiológicos de mudas de Guanandi cultivadas a pleno sol e sob 50 \% de sombreamento, utilizando telas foto-conversoras (azul, preto e vermelho), sendo analisadas no verão e outono de 2012. As mudas cultivadas a pleno sol apresentaram maior qualidade, vigor e acumulo de massa. As telas alteraram o microclima, causando alterações nas trocas gasosas e limitando o desenvolvimento das mudas. A atividade das enzimas antioxidantes mostrou que os tratamentos não causaram estresse oxidativo. O Guanandi apresentou ampla capacidade de aclimatação ao sombreamento e ao sol pleno. Contudo obteve melhores resultados a pleno sol, mesmo sendo considerada espécie tardia a clímax.

Palavras-chave: bioquímica; Calophyllum brasiliensis; ecofisiologia, irradiância.

\section{ACCLIMATION AND PHYSIOLOGY OF GUANANDI SEEDLINGS GROWN UNDER PHOTO- PROTECTIVE SHADE SCREENS}

\begin{abstract}
The luminosity is the most important factor in the growth and development of seedlings. Plants can change their growth model and resource allocation in response to the light environment. Biometric, biochemical and physiological parameters of Guanandi seedlings grown in full sun and under $50 \%$ shade using photo-converter (blue, black and red) screens were evaluated and analyzed in the summer and fall of 2012 The seedlings grown in full sunlight showed higher quality, vigour and mass accumulation. The screens have changed the microclimate, causing changes in gas exchange and limiting the growth of seedlings. The activity of antioxidant enzymes showed that the treatments did not cause oxidative stress. Guanandi presented ample capacity for acclimation to shade and full sun. However performed better in full sun, even been considered late climax species.
\end{abstract}

Keywords: biochemistry; Calophyllum brasiliensis; ecophysiology, irradiance. 
INTRODUÇÃO

A disponibilidade de radiação é o fator básico para o fluxo de energia em sistemas biológicos, sendo determinante para os processos fisiológicos das plantas. Dependendo da capacidade de captação e utilização da luz, as plantas apresentam respostas diferentes e determinantes para a sua sobrevivência, crescimento e adaptação a diferentes ambientais. Por esta razão, os ambientes de luz solar plena ou sombra completa podem inibir os processos fotossintéticos, seja por falta de energia de entrada ou excesso da mesma (ZHANG et al., 2003). Alguns autores sugerem que espécies tolerantes à sombra são mais susceptíveis à fotoinibição e se recuperam em menor extensão que as espécies pioneiras ou dependentes de luz, espécies de clareiras ou ambientes abertos (KRAUSE et al., 2001). Características de crescimento e alocação de biomassa são utilizadas para inferir o grau de tolerância ou de intolerância das espécies à variação na disponibilidade luminosa (SCALON et al., 2002; CARVALHO et al., 2006).

Telas de sombreamento podem afetar as condições ambientais (umidade, sombra/luz, temperatura) e fornecer proteção física as mudas. Podem aumentar a proporção relativa de luz difusa no ambiente e absorver e refletir diferentes bandas espectrais, alterando a qualidade da luz (PÉREZ et al., 2006). A variação da luminosidade pode afetar diretamente os diversos fotorreceptores presentes nas plantas (FOLTA; MARUHNICH, 2007), ocasionando alterações metabólicas e consequentemente influenciando o crescimento e desenvolvimento vegetal (OREN-SHAMIR et al., 2001). Desta forma, a eficiência do crescimento pode ser relacionada à habilidade de adaptação das mudas às condições luminosas do ambiente, sendo o crescimento satisfatório de algumas espécies, em ambientes com baixa ou alta luminosidade, atribuído à capacidade da espécie em ajustar seu modelo de alocação de biomassa e comportamento fisiológico (DIAS-FILHO, 1999). Plantas que crescem a sombra desenvolvem estrutura e aparência diferentes daquelas que crescem em ambiente de luz solar plena (DA MATTA, 2004).

O objetivo deste trabalho foi investigar a plasticidade fenotípica e a aclimatação de mudas de Guanandi, visando testar a hipótese de que alterações nas características espectrais da radiação solar e do microclima podem modificar características estruturais e fisiológicas das plantas, levando a um processo de aclimatação decorrente da plasticidade fenotípica. Para isso foi feita analise 
fisiológica, bioquímica e biométrica de mudas de guanandi cultivadas sob telas de sombreamento (50\%) foto-conversoras (Azul, Vermelha e Preta) e compará-las com as cultivadas a pleno sol.

\section{MATERIAL E MÉTODOS}

O experimento foi desenvolvido no viveiro de mudas do Departamento de Ciências Florestais da Faculdade de Ciências Agronômicas (FCA-UNESP) de Botucatu, no período de Dezembro de 2011 a Junho de 2012. As sementes de Guanandi (Calophyllum brasiliensis) germinaram em casa de germinação a temperatura ambiente $(18-24$ ${ }^{\circ} \mathrm{C}$ ), umidade relativa do ar mantida por volta de $80 \%$ e luminosidade controlada por telas de sombreamento. Após a emergência do primeiro par de folhas as plântulas foram transferidas para tubetes de $250 \mathrm{~cm}^{3}$ com substrato comercial PlantMax Florestal ${ }^{\circledR}$, que é formado por casca de pinus, vermiculita expandida e turfa, na formulação 4:20:6 de NPK, onde permaneceram sob os tratamentos por cerca de 180 dias. Os tratamentos consistiram em sombreamento das mudas por telas foto-conversoras na cor Azul (T1), vermelha (T2) e preta (T3) da Polysack Plastic Industries ${ }^{\circledR}$, e tratamento testemunha a pleno sol (TO). As telas possuem, segundo o fabricante, uma densidade da malha que fornece $50 \%$ de transmitância na região fotossintéticamente ativa.

Tabela 1. Condições ambientais médias fornecidas pelos tratamentos aplicados às mudas de Guanandi no Verão e Outono de 2012. Botucatu-SP, Dados obtidos na câmara de medidas do IRGA.

\begin{tabular}{cccc|ccc} 
& \multicolumn{5}{c}{ Outono } \\
\cline { 2 - 7 } & $\begin{array}{c}\text { Temperatura } \\
\left({ }^{\circ} \mathrm{C}\right)\end{array}$ & $\begin{array}{c}\text { DFFFA }(\mu \mathrm{mol} \\
\left.\text { fótons } \mathrm{m}^{-2} \mathrm{~s}^{-1}\right)\end{array}$ & $\begin{array}{c}\mathrm{Vpd} \\
(\mathrm{Kpa})\end{array}$ & $\begin{array}{c}\text { Temperatura } \\
\left({ }^{\circ} \mathrm{C}\right)\end{array}$ & $\begin{array}{c}\text { DFFFA }(\mu \mathrm{mol} \\
\left.\text { fótons } \mathrm{m}^{-2} \mathrm{~s}^{-1}\right)\end{array}$ & $\begin{array}{c}\text { Vpd } \\
(\mathrm{Kpa})\end{array}$ \\
\cline { 2 - 7 } T0 & 27,6 & 1522 & 1,77 & 21,6 & 1294 & 0,86 \\
T1 & 29,2 & 474 & 2,13 & 23,9 & 273 & 1,19 \\
T2 & 30,0 & 561 & 2,28 & 26,0 & 415 & 1,55 \\
T3 & 30,9 & 546 & 2,32 & 26,0 & 283 & 1,61 \\
\hline
\end{tabular}

As medidas de trocas gasosas foram feitas no segundo par de folhas completamente expandidas aos 90 (Verão) e 180 (Outono) dias de cultivo, sempre no período das 09:00 as 11:00 horas da manhã. Foi utilizado equipamento com sistema aberto de fotossíntese com analisador de $\mathrm{CO}_{2}$ e vapor d'água por radiação infravermelha ("Infra Red Gas Analyser - IRGA", modelo LI6400, LI-COR, Lincoln, NE, EUA). Foram medidas a taxa de assimilação de $\mathrm{CO}_{2}(\mathrm{~A})$, ( $\left.\mu \mathrm{mol} \mathrm{CO}_{2} \mathrm{~m}^{-2} \mathrm{~s}^{-1}\right)$, a condutância estomática (gs) $\left(\mathrm{mol} \mathrm{m} \mathrm{m}^{-2} \mathrm{~s}^{-1}\right)$, a transpiração (E) (mmol $\left.\mathrm{CO}_{2} \mathrm{~m}^{-2} \mathrm{~s}^{-1}\right)$, a concentração interna de $\mathrm{CO}_{2}$ 
(ci) $\left(\mathrm{mmol} \mathrm{CO}_{2} \mathrm{~m}^{-2} \mathrm{~s}^{-1}\right)$ e o déficit de pressão de vapor (Vpd) (Kpa). Da relação entre $(A)$ por (T) foi obtida a eficiência do uso de água (EUA) e da relação entre $(A)$ por (ci) foi obtido à eficiência de carboxilação in vivo da Rubisco (ECR). Antes de medições, o sistema de trocas gasosas foi calibrado utilizando o $\mathrm{CO}_{2}$ e vapor de $\mathrm{H}_{2} \mathrm{O}$ presentes na atmosfera. A Concentração de $\mathrm{CO}_{2}$ no ar foi de $390 \pm 10$ umol. mol ${ }^{-1}$ durante as medições. Para tais medidas foram utilizados a radiação fotossintéticamente ativa (DFFFA) natural presente no ambiente sob as telas no momento das medidas.

Os atributos biométricos foram medidos aos 90 e 180 dias de cultivo. A altura foi medida utilizando uma régua milimétrica e o diâmetro de colo foi medido com um paquímetro digital. Oito plantas de cada tratamento foram coletadas e armazenadas em estufa com circulação forçada de ar a 72 ${ }^{\circ} \mathrm{C}$, onde permaneceram até atingir massa constante, para realizar a pesagem dos órgãos separadamente em balança analítica de precisão. Oito plantas de cada tratamento também foram selecionas para medir a área foliar em um integrador de área, Area Meter, modelo Li-3100 (LI-COR). Para se mensurar a qualidade das mudas e sua posterior sobrevida no campo, utilizou-se o índice de qualidade de Dickson (IQD), que foi determinado em função da massa seca total (PMST), dividido pela relação entre altura da parte aérea $(H)$ e diâmetro do coleto (DC), somado a relação entre massa seca da parte aérea (PMSPA) e da massa seca das raízes (PMSR), por meio da fórmula encontrada em Dickson et al. (1960).

Para a análise bioquímica, folhas foram coletadas as $14: 00$ hs e embrulhadas em papel alumínio e sacos plásticos, sendo imersas em nitrogênio líquido e armazenadas em ultra freezer a -80 ㅇ. A obtenção do extrato bruto se deu através da maceração do material vegetal processado (300 $\mathrm{mg}$ ) em 3,0 $\mathrm{mL}$ de tampão fosfato de potássio $0,1 \mathrm{M}$ com pH 6,8. Após a maceração as amostras foram centrifugadas por 10 minutos a 10000 rpm, sendo então, o sobrenadante coletado em micro tubos. A determinação teor total de proteínas solúveis seguiu o método de Bradford (1976). Posteriormente determinou-se a atividade da enzima peroxidase (POD) seguindo e método de Teisseire e Guy (2000), da catalase (CAT) segundo Peixoto et al. (1999), da superóxido dismutase (SOD) segundo Beauchamp e Fridovich (1971) e para a atividade da nitrato redutase (NR) utilizou-se o método de Streeter e Bosler (1972). Os pigmentos totais foram determinados segundo o método de Sims e Gamon (2002).

\section{RESULTADOS}

As mudas cultivadas a pleno sol apresentaram maior vigor e robustez, indicados pelas taxas de acúmulo de massa e 
crescimento significativamente maiores em relação aos tratamentos sombreados, nas duas estações avaliadas (Tabela 2). As maiorias dos parâmetros apresentaram aumento significativo entre as estações. No verão, as mudas a pleno sol mostraram melhores resultados para a maioria dos atributos, mas com diferença significativa apenas para massa seca de folha e altura. No Outono, a qualidade superior das mudas a pleno sol se acentuou, mostrando superioridade significativa para todos os atributos biométricos avaliados. No verão o IQD não mostrou diferença significativa entre os tratamentos. Contudo no outono as mudas cultivadas a pleno sol que apresentaram um IQD consideravelmente maior. O IQD é apontado como bom indicador da qualidade de mudas, por considerar em seu cálculo a robustez e o equilíbrio da distribuição da biomassa das mudas, sendo ponderados vários parâmetros importantes (FONSECA, 2000).

Tabela 2. Atributos biométricos avaliados em mudas de Guanandi, cultivadas em diferentes luminosidades, no Verão e Outono de 2012. Botucatu-SP

\begin{tabular}{|c|c|c|c|c|c|c|c|c|}
\hline & \multicolumn{4}{|c|}{ Verão } & \multicolumn{4}{|c|}{ Outono } \\
\hline & T0 & T1 & $\mathrm{T} 2$ & T3 & TO & $\mathrm{T} 1$ & $\mathrm{~T} 2$ & T3 \\
\hline $\begin{array}{l}\text { Massa seca de } \\
\text { Raiz (g) }\end{array}$ & $0,44 a B$ & $0,45 \mathrm{aB}$ & $0,41 \mathrm{aB}$ & $0,48 \mathrm{aB}$ & $\begin{array}{c}1,04 \\
\mathrm{aA}\end{array}$ & $\begin{array}{c}0,78 \\
\text { bA }\end{array}$ & $0,81 \mathrm{bA}$ & $0,71 \mathrm{bA}$ \\
\hline $\begin{array}{c}\text { Massa seca de } \\
\text { Caule (g) }\end{array}$ & $0,44 \mathrm{aB}$ & $\begin{array}{l}0,36 \\
a b B\end{array}$ & $\begin{array}{c}0,29 \\
b B\end{array}$ & $\begin{array}{l}0,37 \\
a b B\end{array}$ & $\begin{array}{c}1,03 \\
\mathrm{aA}\end{array}$ & $0,48 \mathrm{cA}$ & $0,63 \mathrm{bA}$ & $0,70 \mathrm{bA}$ \\
\hline $\begin{array}{c}\text { Massa seca de } \\
\text { Folha (g) }\end{array}$ & $\begin{array}{c}0,48 \\
\mathrm{aA}\end{array}$ & $0,39 \mathrm{bA}$ & $0,31 \mathrm{cA}$ & $\begin{array}{l}0,34 \\
\text { bcA }\end{array}$ & $\begin{array}{c}0,84 \\
\mathrm{aA}\end{array}$ & $\begin{array}{c}0,52 \\
b A\end{array}$ & $0,59 \mathrm{bA}$ & $\begin{array}{l}0,66 \\
a b A\end{array}$ \\
\hline $\begin{array}{c}\text { Massa seca total } \\
\text { (g) }\end{array}$ & $1,37 \mathrm{aB}$ & $1,21 \mathrm{aB}$ & $1,02 a B$ & $1,20 \mathrm{aB}$ & $\begin{array}{c}2,92 \\
\mathrm{aA}\end{array}$ & $\begin{array}{c}1,79 \\
b A\end{array}$ & $2,04 \mathrm{bA}$ & $2,08 \mathrm{bA}$ \\
\hline Altura $(\mathrm{cm})$ & $\begin{array}{c}12,92 \\
a B\end{array}$ & $\begin{array}{c}11,10 \\
b B\end{array}$ & $\begin{array}{c}11,47 \\
b B\end{array}$ & $\begin{array}{c}11,36 \\
b B\end{array}$ & $\begin{array}{c}16,43 \\
\mathrm{aA}\end{array}$ & $\begin{array}{c}14,56 \\
b A\end{array}$ & $\begin{array}{c}14,66 \\
b A\end{array}$ & $\begin{array}{c}14,26 \\
b A\end{array}$ \\
\hline $\begin{array}{l}\text { Diâmetro de colo } \\
\qquad(\mathrm{mm})\end{array}$ & $3,69 \mathrm{aB}$ & $3,26 \mathrm{aB}$ & $3,45 a B$ & $3,32 \mathrm{aA}$ & $\begin{array}{c}4,72 \\
\mathrm{aA}\end{array}$ & $\begin{array}{c}3,81 \\
b A\end{array}$ & $4,00 \mathrm{bA}$ & $4,09 \mathrm{bA}$ \\
\hline Área Foliar $\left(\mathrm{cm}^{2}\right)$ & $\begin{array}{c}47,37 \\
\mathrm{aB}\end{array}$ & $\begin{array}{c}40,62 \\
a b A\end{array}$ & $\begin{array}{c}28,91 \\
b B\end{array}$ & $\begin{array}{c}30,33 \\
a b B\end{array}$ & $\begin{array}{c}69,76 \\
\mathrm{aA}\end{array}$ & $\begin{array}{c}46,39 \\
b A\end{array}$ & $\begin{array}{c}56,00 \\
a b A\end{array}$ & $\begin{array}{c}53,14 \\
a b A\end{array}$ \\
\hline IQD & $0,15 \mathrm{aB}$ & $0,16 \mathrm{aB}$ & $0,14 \mathrm{aB}$ & $0,17 \mathrm{aB}$ & $\begin{array}{c}0,39 \\
\mathrm{aA}\end{array}$ & $\begin{array}{c}0,24 \\
b A\end{array}$ & $0,28 \mathrm{bA}$ & $0,26 \mathrm{bA}$ \\
\hline
\end{tabular}

*Médias seguidas da mesma letra minúscula diferem significativamente quanto aos tratamentos e por letras maiúsculas quanto à estação do ano, segundo Teste de Tukey, a 5\% de probabilidade (SISVAR).

As mudas cultivadas a pleno sol apresentaram maiores taxas de assimilação liquida de $\mathrm{CO}_{2}(\mathrm{~A})$ em relação ás espécies sombreadas nas duas estações avaliadas. No Verão, as telas de sombreamento alteraram - micro clima ao redor das mudas diminuindo a DFFFA e provocando aumento na temperatura média e no déficit de pressão de vapor em relação ao pleno sol. No Outono todos os tratamentos apresentaram aumento significativo nos valores de (A). As mudas a pleno sol apresentaram valores de (A) 
consideravelmente maiores, juntamente com uma alta eficiência de carboxilação da Rubisco (ECR) e maior eficiência do uso da água (EUA) em relação aos dados observados no verão. (Tabela 3). No entanto as mudas de
Guanandi não apresentaram diferenças significativas quanto aos teores de pigmentos foliares (Tabela 4) entre os tratamentos e entre as estações do ano.

Tabela 3. Análise de trocas gasosas em mudas de Guanandi, cultivadas em diferentes luminosidades, no Verão e Outono de 2012. Botucatu-SP

\begin{tabular}{|c|c|c|c|c|c|c|c|c|}
\hline & \multicolumn{4}{|c|}{ Verão } & \multicolumn{4}{|c|}{ Outono } \\
\hline & TO & $\mathrm{T} 1$ & $\mathrm{~T} 2$ & T3 & TO & $\mathrm{T} 1$ & $\mathrm{~T} 2$ & T3 \\
\hline$A\left({ }_{2} \mathrm{~s}^{-1}\right)$ & $9,05 \mathrm{aB}$ & $5,50 \mathrm{bA}$ & $5,84 \mathrm{bB}$ & $4,81 \mathrm{bB}$ & $\begin{array}{c}15,22 \\
a A\end{array}$ & $7,35 \mathrm{bA}$ & $8,37 \mathrm{bA}$ & 8,76 bA \\
\hline $\begin{array}{c}\text { gs }(\mathrm{mmol} \\
\left.\mathrm{m}^{-2} \mathrm{~s}^{-1}\right)\end{array}$ & $0,11 \mathrm{aB}$ & $0,05 \mathrm{bA}$ & $0,05 \mathrm{bA}$ & $0,06 \mathrm{bA}$ & 0,07 aA & 0,07 aA & 0,05 aA & $0,08 \mathrm{aA}$ \\
\hline $\mathrm{Ci}(\mathrm{ppm})$ & $\begin{array}{c}228,25 \\
a B\end{array}$ & $\begin{array}{c}193,51 \\
a A\end{array}$ & $\begin{array}{c}162,39 \\
\mathrm{aA}\end{array}$ & $\begin{array}{c}239,06 \\
a A\end{array}$ & $\begin{array}{c}132,66 \\
b A\end{array}$ & $\begin{array}{c}198,09 \\
\text { bA }\end{array}$ & $\begin{array}{c}126,90 \\
b A\end{array}$ & $\begin{array}{c}262,77 \\
a A\end{array}$ \\
\hline$E\left(\underset{2}{2} \mathrm{~s}^{-1}\right)$ & $2,29 a B$ & $1,24 \mathrm{bA}$ & $1,40 \mathrm{bA}$ & $\begin{array}{l}1,63 \\
a b A\end{array}$ & $0,79 \mathrm{bA}$ & $\begin{array}{l}0,95 \\
a b A\end{array}$ & $\begin{array}{l}0,95 \\
a b A\end{array}$ & $1,61 \mathrm{aA}$ \\
\hline EUA & $3,94 \mathrm{aB}$ & $4,42 \mathrm{aA}$ & $4,15 \mathrm{aA}$ & $2,95 \mathrm{aA}$ & $\begin{array}{c}19,23 \\
a A\end{array}$ & $7,68 \mathrm{bA}$ & $8,76 \mathrm{bA}$ & $5,43 \mathrm{bA}$ \\
\hline ECR & $0,03 a B$ & $0,02 \mathrm{aA}$ & $0,04 \mathrm{aA}$ & $0,02 \mathrm{bA}$ & 0,11 aA & $0,03 \mathrm{bA}$ & $\begin{array}{l}0,06 \\
a b A \\
\end{array}$ & $0,03 \mathrm{bA}$ \\
\hline
\end{tabular}

*Médias seguidas da mesma letra minúscula diferem significativamente quanto aos tratamentos e por letras maiúsculas quanto à estação do ano, segundo Teste de Tukey, a 5\% de probabilidade (SISVAR).

A análise bioquímica das mudas mostrou que as atividades das enzimas POD, SOD e CAT não diferiram significativamente entre os tratamentos e entre as estações, nem o tratamento a pleno sol causou estresse oxidativo (Tabela 4). A atividade da enzima nitrato redutase apresentou um valor significativamente maior no tratamento a pleno sol e no tratamento com a tela vermelha nas duas estações em relação aos outros tratamentos. 
Tabela 4. Análise Bioquímica das mudas de Guanandi, cultivadas em diferentes luminosidades, no Verão e Outono de 2012. Botucatu-SP

\begin{tabular}{|c|c|c|c|c|c|c|c|c|}
\hline & \multicolumn{4}{|c|}{ Verão } & \multicolumn{4}{|c|}{ Outono } \\
\hline & T0 & $\mathrm{T} 1$ & $\mathrm{~T} 2$ & T3 & TO & $\mathrm{T} 1$ & $\mathrm{~T} 2$ & T3 \\
\hline NR $(\mu \mathrm{g} / \mathrm{min} / \mathrm{g} \mathrm{MF})$ & $6,04 \mathrm{aA}$ & $2,70 \mathrm{cA}$ & $4,67 \mathrm{bA}$ & $2,90 \mathrm{cA}$ & $5,13 \mathrm{aB}$ & $2,73 \mathrm{bA}$ & $5,14 \mathrm{aA}$ & $3,02 \mathrm{bA}$ \\
\hline $\begin{array}{l}\text { CAT }(\mathrm{m} / \mu \mathrm{g} \\
\text { proteína) }\end{array}$ & 0,27 aA & $0,34 \mathrm{aA}$ & 0,29 aA & $0,27 \mathrm{aA}$ & $0,32 \mathrm{aA}$ & $0,28 \mathrm{aA}$ & $0,18 \mathrm{aA}$ & $0,23 \mathrm{aA}$ \\
\hline $\begin{array}{l}\text { SOD (U/ } \mu \mathrm{g} \\
\text { proteína) }\end{array}$ & $1,12 \mathrm{bA}$ & $\begin{array}{l}1,52 \\
a b A\end{array}$ & $1,53 \mathrm{aA}$ & $\begin{array}{l}1,49 \\
a b A\end{array}$ & $1,21 \mathrm{aA}$ & $1,34 \mathrm{aA}$ & $1,10 \mathrm{aB}$ & $1,18 \mathrm{aB}$ \\
\hline $\begin{array}{l}\mathrm{POD}(\mu \mathrm{mol} / \mathrm{min} / \mathrm{mg} \text {. } \\
\text { prot) }\end{array}$ & $\begin{array}{c}20,28 \\
\mathrm{Aa}\end{array}$ & $\begin{array}{c}18,68 \\
\mathrm{Aa}\end{array}$ & $\begin{array}{c}16,29 \\
\mathrm{Aa}\end{array}$ & $9,49 \mathrm{Ba}$ & $\begin{array}{c}14,91 \\
A b\end{array}$ & $\begin{array}{c}18,46 \\
\mathrm{Aa}\end{array}$ & $8,55 \mathrm{bB}$ & $6,15 \mathrm{bA}$ \\
\hline Clorofila a $(\mu \mathrm{mol} / \mathrm{g})$ & $\begin{array}{l}335 \\
37 a A\end{array}$ & $\begin{array}{l}362,77 \\
a A\end{array}$ & $\begin{array}{l}225,08 \\
b A\end{array}$ & $\begin{array}{c}401,89 \\
\mathrm{aA}\end{array}$ & $\begin{array}{c}301,03 \\
a A\end{array}$ & $\begin{array}{c}298,67 \\
a A\end{array}$ & $\begin{array}{c}265,19 \\
a A\end{array}$ & $\begin{array}{c}304,14 \\
a B\end{array}$ \\
\hline Clorofila b ( $\mu \mathrm{mol} / \mathrm{g})$ & $\begin{array}{c}145,39 a \\
\text { bA }\end{array}$ & $\begin{array}{c}170,48 \\
\mathrm{aA}\end{array}$ & $\begin{array}{c}102,69 \\
b A\end{array}$ & $\begin{array}{c}182,09 \\
a A\end{array}$ & $\begin{array}{c}131,48 \\
\mathrm{aA}\end{array}$ & $\begin{array}{c}133,31 \\
a A\end{array}$ & $\begin{array}{c}131,07 \\
\mathrm{aA}\end{array}$ & $\begin{array}{c}165,55 \\
\mathrm{aA}\end{array}$ \\
\hline $\begin{array}{c}\text { Antocianinas } \\
(\mu \mathrm{mol} / \mathrm{g})\end{array}$ & $\begin{array}{l}0,33 \\
a b A\end{array}$ & $0,24 \mathrm{bB}$ & $0,42 \mathrm{aA}$ & $\begin{array}{c}0,34 a b \\
A\end{array}$ & $\begin{array}{l}0,41 \\
a b A\end{array}$ & $\begin{array}{l}0,34 \\
a b A\end{array}$ & $0,30 \mathrm{bB}$ & $0,44 \mathrm{aA}$ \\
\hline $\begin{array}{l}\text { Carotenoides } \\
(\mu \mathrm{mol} / \mathrm{g})\end{array}$ & $\begin{array}{c}545,49 \\
\mathrm{aA}\end{array}$ & $\begin{array}{l}549,61 \\
\mathrm{aA}\end{array}$ & $\begin{array}{c}384,86 \\
\text { bA }\end{array}$ & $\begin{array}{c}644,98 \\
\mathrm{aA}\end{array}$ & $\begin{array}{c}455,75 \\
\mathrm{aA}\end{array}$ & $\begin{array}{c}471,04 \\
\mathrm{aA}\end{array}$ & $\begin{array}{c}473,72 \\
\mathrm{aA}\end{array}$ & $\begin{array}{c}389,98 \\
a B\end{array}$ \\
\hline
\end{tabular}

*Médias seguidas da mesma letra minúscula diferem significativamente quanto aos tratamentos e por letras maiúsculas quanto à estação do ano, segundo Teste de Tukey, a 5\% de probabilidade (SISVAR).

\section{DISCUSSÃO}

Embora o Guanandi seja considerado uma espécie tardia a clímax na escala de sucessão ecológica (tolerante ao sombreamento), observou-se alta capacidade de aclimatação ao ambiente de maior luminosidade, onde os indivíduos tiverem maior taxa de acumulo de massa $\left(0,2 \mathrm{~g} \mathrm{dia}^{-1}\right)$, em relação aos indivíduos crescendo nos tratamentos sombreados $\left(0,1 \mathrm{~g} \mathrm{dia}^{-1}\right)$. Essa capacidade de aclimatação do Guanandi já havia sido registrada antes por trabalhos de Lorenzi (1992) e Duffecky e Fossati (2009).

Um dos efeitos dos tratamentos de sombreamento no cultivo de mudas de Guanandi foi à alteração causada no microclima ao redor das mudas. Os microclimas nos tratamentos sombreados induziram alterações significativas nas trocas gasosas, como o aumento do fechamento estomático causado pelo aumento do déficit de pressão de vapor, associado a maior temperatura e menor luminosidade em relação ao pleno sol. A literatura afirma que um aumento do DPV de 1 para $2 \mathrm{kPa}$, em função do aumento da temperatura, pode causar quedas de até $50 \%$ na taxa de fotossíntese em mudas de algumas espécies arbóreas, devido á limitação estomática (HABERMANN et al., 2003). Outro fator abiótico que sofre influencia é o vento, que tem sua velocidade e circulação diminuídas, fato que pode afetar a temperatura (STAMPS, 1994), aumentar a umidade relativa do ar (ELAD et al., 2007) e as concentrações de gases resultantes de 
reduções de mistura de ar (ROSENBERG et al., 1983). Essas mudanças podem afetar a transpiração, fotossíntese, respiração e outros processos fisiológicos.

No outono, o fator que parece mais ter influenciado negativamente no desempenho das mudas sombreadas foi à baixa radiação. Nessa estação as mudas cultivadas a pleno sol apresentaram ECR, EUA e (A) duas vezes maior que as sombreadas, concordando com Larcher (2006) que afirma que espécies crescendo sob radiação solar plena, geralmente apresentam maiores valores de capacidade fotossintética e eficiência de carboxilação. No outono a EUA aumentou em todos os tratamentos com relação ao verão, fato relacionado à diminuição nas taxas de transpiração apresentadas no mesmo período. Um fator que pode ter ajudado na aclimatação das mudas foi ser seu alto teor de pigmentos carotenoides, que possuem função fotoprotetora na fotossíntese (HENDRY; PRICE, 1993).

\section{CONCLUSÃO}

Os resultados do trabalho apontam que o Guanandi pode responder plasticamente às variações em seu ambiente, sendo capaz de exibir um crescimento superior em altas condições de luminosidade. Entretanto, os resultados não podem ser generalizados, pois alguns autores citam que a influência da qualidade da radiação sobre o crescimento e o desenvolvimento de mudas está associada à espécie vegetal em questão. Assim, pode ser corroborada a hipótese de que alterações nas características espectrais da radiação solar e do microclima podem modificar características estruturais e fisiológicas das plantas, levando a um processo de aclimatação decorrente da plasticidade fenotípica, observando-se sempre a individualidade de cada espécie e o ambiente no qual a planta se encontra.

\section{REFERÊNCIAS}

BEAUCHAMP, C.; FRIDOVICH, I. Superoxide dismutase: improved assays and applicable to acrylamide gels. Analytical Biochemistry, new York, v.44, n.1, p. 276-287, 1971. http://dx.doi.org/10.1016/00032697(76)90527-3

BRADFORD, M.M. A rapid and sensitive method for the quantifications of microgram quantities of protein utilizing the principle of protein-dye binding. Analitycal Biochemistry, Alexandria, v.72, p. 248-254, 1976.

CARVALHO, N.O.S.; PELACANI, C.R.; RODRIGUES, M.O.S.; CREPALDI, I.C. Initial growth of licuri plants (Syagruscoronata (Mart.) Becc.) under diferente light intensity. Revista Árvore, n.30, p.351-357, 2006. http://dx.doi.org/10.1590/S0100$\underline{67622006000300005}$

DA MATTA, F.M. Ecophysiological constraints on the production of shaded and unshaded coffee: a review. Field Crops Research, v.86, p.99-114, 2004. http://dx.doi.org/10.1016/j.fcr.2003.09.001 
DIAS-FILHO, M. B. Physiological responses of two tropical weeds to shade. I. Growth and biomass allocation. Pesquisa Agropecuária Brasileira, 34 (6): 945-952, 1999.

DICKSON, A.; LEAF, A.L.; HOSNER, J.F. Quality appraisal of white spruce and white pine seedling stock in nurseries. For. Chron., n.36, p.10-13, 1960.

\section{http://dx.doi.org/10.5558/tfc36010-1}

DUFFECKY, M.D.; FOSSATI, L.C. Avaliação da adaptação de Calophyllum brasiliensis Cambess. (Guanandi), Família Clusiaceae, no Planalto Norte Catarinense. Ágora: R. Divulg. Cient., n.16, p.2, 2009.

ELAD, Y.Y.; MESSIKA, M.; BRAND, D.R.; DAVID, A. Effect of colored shade nets on pepper powdery mildew (Leveillula taurica). Phytoparasitica, v.35, p.285-299, 2007. http://dx.doi.org/10.1007/BF02981163

FOLTA, K.M.; MARUHNICH, S.A. Green light: a signal to slow down or stop. J. Expt. Bot., n.58, p.3099-3111, 2007. http://dx.doi.org/10.1093/jxb/erm130

FONSECA, E. P. Padrão de qualidade de mudas de Trema mícrantha (L.) Blume, Cedrela fissilis Veli. E Aspidosperma polyneuron Müll Arg. Produzidas sob diferentes períodos de sombreamento. 2000. 133f. Tese (Doutorado em Agronomia) Universidade Estadual Paulista, Jabotical SP. $\quad$ http://dx.doi.org/10.1007/978-94-011$\underline{1494-3}$

HENDRY, G.A.F.; PRICE, A.H. Stress indicators: chlorophylls and carotenoids. In: HENDRY, G.A.F.; GRIME J.P. (Eds.) Methods in comparative plant ecology. London: Chapman \& Hall, 1993. p.148-152.

KRAUSE, G.H.; KOROLEVA, O.Y.; DALLING, J.W.; WINTER, K. Acclimation of tropical tree seedlings to excessive light in simulated treefall gaps. Plant, Cell and Environment, v.24, p.1345-1352,

2001. http://dx.doi.org/10.1046/i.0016-

8025.2001.00786.x

HABERMANN, G.; MACHADO, E.C.; RODRIGUES, J.D.; MEDINA, C.L. Gas exchange rates at different vapor pressure deficits and water relations of 'Pera' sweet orange plants with citrus variegated chlorosis. Scientia Horticulturae, v.98, p.233-245, 2003. http://dx.doi.org/10.1016/S03044238(02)00228-5

LARCHER, W. Ecofisiologia Vegetal. São Carlos: RiMa, 550p., 2006.

LORENZI, H. Árvores brasileiras: manual de identificação e cultivo de plantas arbóreas do Brasil. 4. ed. Nova Odessa: Instituto Plantarum, 2002.

OREN-SHAMIR, M.; GUSSAKOVSKY, E.E.; SHPIEGEL, E.; NISSIM-LEVI, A.; RATNER, K.; OVADIA, R.; GILLER, Y.E.; SHAHAK, Y. Coloured shade nets can improve the yield and quality of green decorative branches of Pittosporum variegatum. J. Hort. Sci. Biotechnol., n.76, p.353-361, 2001.

PEIXOTO, H.P.P. et al. Aluminium effects on lipid peroxidation and the activities of enzimes of oxidative metabolism in sorghum. Revista Brasileira de Fisiologia Vegetal. Londrina, v.11, n.3, p. 137-143, 1999.

PÉREZ, M.; PLAZA, B.M.; JIMÉNEZ, S.; LAO, M.T.; BARBERO, J.; BOSCH., J.L. The radiation spectrum through ornamental net houses and its impact on the climate generated. Acta Hort., v.719, p.631-636, 2006.

ROSENBERG, N. J.; BLAD, B. L.; VERMA, S. B. Microclimate: the biological environment. 2.ed. New York: John Wiley \& Sons, 1983.

SCALON, S.P.Q.; MUSSURY, R.M.; VERALDO, $F$. Crescimento inicial de mudas de espécies florestais nativas sob diferentes níveis de sombreamento. Revista Árvore, n.26, p.1-5, 2002. 
SIMS, D. A.; GAMON, J. A. Relationships between leaf pigment content and spectral reflectance across a wide range of species, leaf structures and developmental stages. Remote Sensing of Environment. v.81, p.337354, 2002. http://dx.doi.org/10.1016/S00344257(02)00010-X

STAMPS, R. H. Evapotranspiration and nitrogen leaching during leather leaf fern production in shadehouses. SJRWMD Spec. Publ., SJ94-SP10, 1994.

STREETER, J.G.; BOSLER, M.E. Comparison of in vitro and in vivo assays for nitrate reductase in soybean leaves. Plant Physiology, Minneapolis, v.49, n.3, p.448, 1972. http://dx.doi.org/10.1104/pp.49.3.448

TEISSEIRE , H.; GUY, V. Copper-induced changes in antioxidante enzymes activities in fronds of duckweed (Lemna minor). Plant Science, Lumerick, v.153, n.1, p. 65-72, 2000.

ZHANG S.; MA K.; CHEN L. 2003. Response of photosynthetic plasticity of Paeonia suffruticosa to changed light environments. Environ. Exp. Bot. 49:121-133. http://dx.doi.org/10.1016/S0098-

8472(02)00063-1 